[Article]

\title{
乳液聚合法制备红霉素分子印迹聚合物微球及其吸附性能
}

\author{
赵 娜 ${ }^{*}$ 胡小玲 管 萍 宋任远 田 甜 张向荣
}

(西北工业大学理学院, 教育部空间应用物理与化学重点实验室, 西安 710072)

\begin{abstract}
摘要: 以大环内酯类抗生素红霉素(EM)为模板分子, 甲基丙烯酸(MAA)为功能单体, 乙二醇二甲基丙烯酸酯 (EDMA)为交联剂, 十二烷基苯磺酸钠(SBS)为乳化剂, 采用乳液聚合法制备了粒径均匀的分子印迹聚合物微 球(EM-MIPMs). 通过核磁共振氢谱('H NMR)、紫外光谱和傅里叶变换红外(FTIR)光谱对模板分子和功能单体 形成的复合物进行了研究, 结果表明 EM 与 MAA之间的相互作用力为氢键作用. 利用扫描电镜(SEM)、热重分 析(TGA)仪对 EM-MIPMs 的形貌和热稳定性进行表征, 结果显示 EM-MIPMs 为均匀规整的球型, 平均粒径为 $4.24 \mu \mathrm{m}$, 且有良好的热稳定性. 同时采用动力学, 平衡吸附和选择性吸附实验对其吸附性能进行研究. 动力学 研究结果表明, EM-MIPMs 的吸附速率符合准二级动力学方程. 利用 Langmuir 和 Freundlich 吸附等温方程分 别分析了 EM-MIPMs 的平衡吸附数据, 结果表明, EM-MIPMs 对红霊素有良好的结合性能, 其吸附过程符合 Langmuir 吸附模型, 饱和吸附量为 $0.242 \mathrm{mmol} \cdot \mathrm{g}^{-1}$. EM-MIPMs 的选择识别性能利用固相萃取法来考察, 研究 表明 EM-MIPMs 有着良好的特异识别选择性.
\end{abstract}

关键词: 红霉素; 分子印迹微球; 吸附; 结合性能, 识别性能 中图分类号: $0647 ; 0641$

\section{Preparation of Erythromycin-Imprinted Polymeric Microspheres by Emulsion Polymerization and Their Adsorption Properties}

\author{
ZHAO Na* HU Xiao-Ling GUAN Ping SONG Ren-Yuan TIAN Tian \\ ZHANG Xiang-Rong \\ (The Key Laboratory of Space Applied Physics and Chemistry, Ministry of Education, School of Natural and Applied Science, \\ Northwestern Polytechnical University, Xi'an 710072, P. R. China)
}

\begin{abstract}
Uniform molecularly imprinted polymeric microspheres (EM-MIPMs) were prepared by emulsion polymerization using erythromycin as the template molecule, methacrylic acid (MAA) as the functional monomer, and ethylene glycol dimethacrylate (EDMA) and sodium dodecylbenzene sulfonate (SBS) as the cross-linker and emulsifier, respectively. The obtained erythromycin-MAA complexes were characterized using ultraviolet (UV) absorption spectroscopy, Fourier-transform infrared (FTIR) spectroscopy, and ${ }^{1} \mathrm{H}$ nuclear magnetic resonance (NMR) spectroscopy. The results showed that erythromycin-MAA complexes were obtained by cooperative hydrogen-bonding interactions. The surface features and thermal stability of the EM-MIPMs were investigated using scanning electron microscopy (SEM) and thermal gravimetric analysis (TGA). The average diameter of the EM-MIPMs was $4.24 \mu \mathrm{m}$, larger than non-imprinted polymeric microspheres. They exhibited excellent thermal stability. Kinetic, equilibrium adsorption, and selectivity adsorption experiments (solid-phase extraction) were used to evaluate the binding properties and molecule recognition characteristics of EM-MIPMs for erythromycin. The experimental kinetic data were well described by a pseudo-second-order kinetic model. Erythromycin binding was examined using the Langmuir and Freundlich isotherm models. The EM-MIPMs had an excellent affinity for erythromycin. The equilibrium experimental data for the EM-MIPMs fitted the Langmuir
\end{abstract}

Received: September 18, 2013; Revised: November 25, 2013; Published on Web: November 27, 2013.

"Corresponding author. Email: zhaohx0906@126.com; Tel: +86-29-88431639.

The project was supported by the National Natural Science Foundation of China (21174111).

国家自然科学基金(21174111)资助项目

(C) Editorial office of Acta Physico-Chimica Sinica 
isotherm well, and the binding amount reached $0.242 \mathrm{mmol} \cdot \mathrm{g}^{-1}$. Furthermore, solid-phase extraction experiments demonstrated that the EM-MIPMs had a higher affinity for the target molecules than for roxithromycin and erythromycin ethylsuccinate.

Key Words: Erythromycin; Molecularly imprinted microsphere; Adsorption; Binding property; Recognition property

\section{1 引言}

分子印迹技术是一种制备对特定模板分子具 有特异性识别性能的聚合物材料的技术. ${ }^{1-4}$ 通过该 技术制得的分子印迹聚合物与生物抗体相比, 具有 高度的选择性、化学和机械稳定性以及可重复使用 等优点, 在色谱、手性分离、6 固相萃取、 ${ }^{7}$ 模拟酶催 化、 ${ }^{8}$ 药物控释 ${ }^{9}$ 等领域具有广阔的应用前景. 分子印 迹微球具有制备简单、识别效率高、粒径分布均匀、 便于功能设计等优点, 近些年成为分子印迹技术研 究的热点之一, 其中乳液聚合方法是制备聚合物微 球常用的方法之一. ${ }^{10,11}$ 此外, 基于分子印迹的特异 识别性, 有望克服样品含量低、取样难等不利因素, 为痕量样品的富集及分离提供了一种重要途径. ${ }^{12}$

从人类发现青霉素开始, 抗生素便成为了化学 和医药学领域研究的重点. 随着人们对抗生素残留 危害的逐渐了解, 近年来抗生素也成为分子印迹领 域研究的热点. ${ }^{13,14}$ 作为常用抗生素之一的大环内酯 类抗生素是近年来广泛用于临床及农业生产中的 一类具有共同化学结构和相似抗菌作用的抗菌药. 其中红霉素(EM)是一种十四元环大环内酯抗生素, 主要对革兰氏阳性细菌具有抗菌性, 其分子量较 大, 结构复杂, 使得分子印迹十分困难, 因此如何利 用分子印迹技术实现红霉素分子的快速、高效分离 成为研究的难点.

关于抗生素分子印迹聚合物微球的制备近年 来已有报道, ${ }^{15,16}$ 其主要应用于色谱、固相萃取和传 感器等领域. 而目前红霉素分子印迹聚合物微球的 相关研究相对较少. 其中雷建都研究组分别通过悬 浮聚合法 ${ }^{17}$ 和沉淀聚合法 ${ }^{18}$ 成功制备了红霉素分子 印迹聚合物微球和纳米微球, 但由悬浮聚合法制备 的微球对红霉素的最大结合量较低, 且印迹微球的 粒径分布较宽; 而由沉淀聚合法合成的红霉素分子 印迹聚合物纳米微球虽然吸附量有了较大提高, 但 未对其印迹机理进行深入研究. 本研究组主要从事 大环内酯类抗生素的分子印迹研究, ${ }^{19-21}$ 如红霉素、 罗红霉素等, 为大环内酯类抗生素在水相中的识
别、分离和分析提供了有效的方法. 本文首次采用 乳液聚合法, 以甲基丙烯酸为功能单体制备得到了 粒径均匀且识别性能良好的红霉素分子印迹聚合 物微球(EM-MIPMs). 利用紫外光谱, 傅里叶变换红 外(FTIR)光谱和核磁共振氢谱深入探讨了模板分子 与功能单体之间的相互作用力, 并通过 Langmuir 和 Freundlich 吸附等温方程以及准一级和准二级动力 学方程分别研究了 EM-MIPMs 的吸附性能, 同时采 用固相萃取的方法考察其选择识别性能.

\section{2 实验部分}

\section{1 主要仪器与试剂}

傅里叶变换红外光谱仪(WQF-310, 日本岛津公 司); 紫外-可见光分光光度计(UV-2550, 日本岛津公 司); 环境扫描电镜(Quanta 200, 荷兰 Philips-FEI 公 司); 热重分析仪(Q600 SDT, 美国 TA 仪器公司); 数 显水浴恒温振荡器(SHZ-C, 金坛市江南仪器厂); 数 控超声波清洗器(KQ-100DE, 昆山市超声仪器有限 公司).

红霉素(纯度为 $93 \%$, 利君集团镇江制药有限公 司); 罗红霉素(roxithromycin (RM) 纯度为 $95 \%$, 中国 药品生物制品检定所); 琥乙红霉素 (erythromycin ethylsuccinate (EEs), AR, Ruibio Germany); 乙二醇 二甲基丙烯酸酯(EDMA, 纯度为 $97 \%$, 烟台云开化 工有限公司); 十二烷基苯磺酸钠(SBS, AR), 甲基丙 烯酸(MAA, AR), 过硫酸钾 (KPS, AR)及氯仿 (AR), 西安化学试剂厂; 其余试剂均为分析纯.

\section{2 红霉素分子印迹聚合物微球的制备}

将 $1 \mathrm{mmol} \mathrm{EM}$ 模板分子和 $4 \mathrm{mmol}$ 功能单体 MAA 溶解于 $10 \mathrm{~mL}$ 氯仿溶液中, 加入 $15 \mathrm{mmol}$ 交联 剂(EDMA), 超声使其形成均匀溶液. 再将此混合溶 液中缓慢加入到 $100 \mathrm{~mL}$ 的 $10 \% \mathrm{SBS}$ 水溶液中, 摚 拌使其充分混合形成白色的乳液. 通氮气 $15 \mathrm{~min}$, 升 温至 $60^{\circ} \mathrm{C}$, 然后缓慢加入引发剂 KPS $77.04 \mathrm{mg}$, 最 后在氮气氛围中聚合 $12 \mathrm{~h}$, 冷却至室温, 得到 EMMIPMs. 依次用 $10 \%$ 乙醇水溶液、 $10 \%$ 乙酸溶液、二 


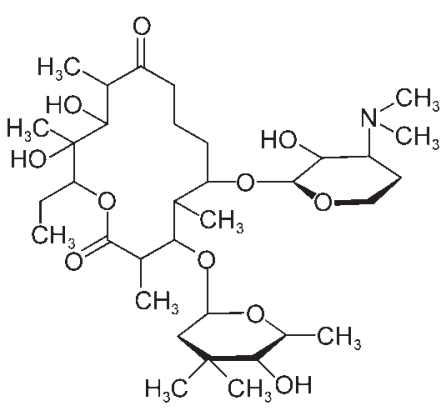

erythromycin

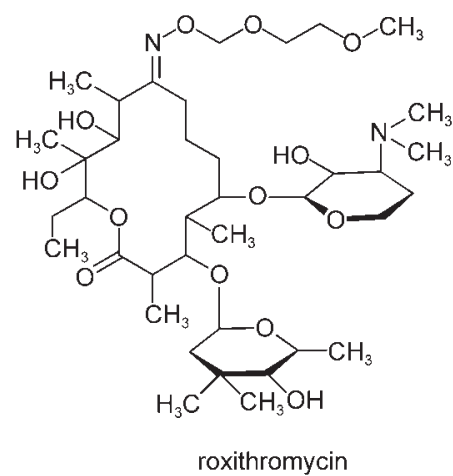

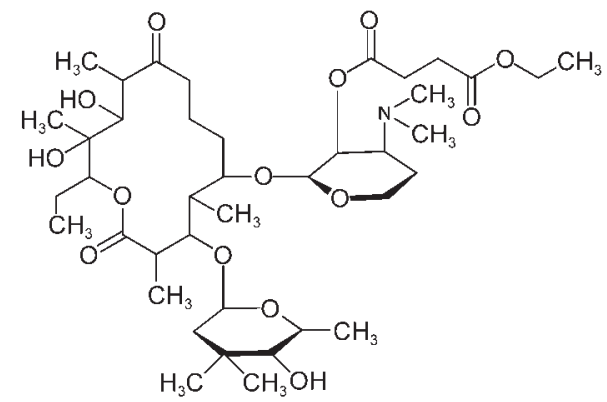

erythromycin ethylsuccinate

图 1 红霉素(EM)、罗红霉素(RM)和琥乙红霉素(EEs)的化学结构式

Fig.1 Chemical structures of erythromycin (EM), roxithromycin (RM), and erythromycin ethylsuccinate (EEs)

次蒸馏水振荡洗脱, 直到用紫外-可见分光光度计 (在磷酸存在条件下吸收(波长为 $483 \mathrm{~nm}$ ) 检测不出 $\mathrm{EM}$ 为止. 最后, 将制备的 EM-MIPMs 于 $60{ }^{\circ} \mathrm{C}$ 真空 干燥至恒重 $(3.38 \mathrm{~g})$ 备用, 聚合产率为 $83.5 \%$.

非印迹聚合物微球(NMIPMs)的制备方法同上, 但在制备过程中不加入模板分子 EM.

\section{3 红霉素分子印迹聚合物微球的吸附性能}

\subsection{1 静态平衡吸附}

准确称取多份一定量的 EM-MIPMs (每份约 $100 \mathrm{mg}$ ), 置于 $50 \mathrm{~mL}$ 锥形瓶中, 然后依次加入 10 $\mathrm{mL}$ 浓度为 $1.0-10.0 \mathrm{mmol} \cdot \mathrm{L}^{-1}$ 的 $\mathrm{EM}$ 的 $20 \%$ (体积 分数)乙腈/水溶液, 于 $25^{\circ} \mathrm{C}$ 下振荡吸附 $8 \mathrm{~h}$, 离心分 离后, 在磷酸存在条件下, 采用紫外分光光度计测 定上清液中 EM 的浓度, 根据吸附前后溶液中 EM 的浓度变化计算EM-MIPMs 的吸附量, 如公式(1)所 示.

$$
Q=\left(C_{0}-C_{\mathrm{e}}\right) V / m
$$

式中 $Q$ 为吸附量 $\left(\mathrm{mmol} \cdot \mathrm{g}^{-1}\right) ; C_{0}$ 为吸附前 $\mathrm{EM}$ 的浓度 $\left(\mathrm{mmol} \cdot \mathrm{L}^{-1}\right) ; C_{\mathrm{e}}$ 为吸附后溶液中 $\mathrm{EM}$ 的浓度 $(\mathrm{mmol} \cdot$ $\left.\mathrm{L}^{-1}\right) ; m$ 为 MIPMs 或 NMIPMs 的质量 $(\mathrm{g}) ; V$ 为吸附液 的体积 $(\mathrm{mL})$. 平行测定三次, 取平均值.

NMIPMs 对 EM 的吸附操作步骤同上.

\subsection{2 吸附动力学研究}

为了研究 EM-MIPMs 的结合动力学, 分别称取 多份 $100 \mathrm{mg}$ EM-MIPMs 和 NMIPMs 置于锥形瓶 中, 加入 $10 \mathrm{~mL}$ 浓度为 $8.0 \mathrm{mmol} \cdot \mathrm{L}^{-1}$ 的红霉素溶液, $25^{\circ} \mathrm{C}$ 振荡吸附, 每隔 $10 \mathrm{~min}$ 取样测定 EM-MIPMs 对 EM 的吸附量, 以研究 EM-MIPMs 对 EM 的吸附 速率.

\section{4 红霉素分子印迹聚合物微球的识别性能}

为研究 EM-MIPMs 印迹聚合物对模板分子的 特异识别性能, 将选择罗红霉素和琥乙红霉素作为
竞争吸附物, 三种吸附物同属于大环内酯类分子, 虽然三者分子结构类似, 但其疗效差异较大, 其化 学结构如图 1 所示.

称取 $1.08 \mathrm{~g}$ EM-MIPMs 装入内径为 $10 \mathrm{~mm}$ 的固 相萃取柱中, 其中填充柱的床体积(BV)为 $2 \mathrm{~mL}$, 分 别配制浓度为 $1 \mathrm{mmol} \cdot \mathrm{L}^{-1}$ 的 $\mathrm{EM} 、 \mathrm{RM}$ 和 EEs 三种 $20 \%$ (体积分数)乙腈/水溶液, 以 $2 \mathrm{BV} \cdot \mathrm{h}^{-1}$ 的流速逆 流通过固相萃取柱, 并以 $2 \mathrm{BV}$ 的间隔收集流出液, 测定流出液中待测物质的浓度, 并绘制出动态结合 曲线, 利用流出液的浓度与床体积数, 计算 EMMIPMs 对三种待测物质的泄漏吸附量与饱和吸附 量.

采用相同的方法测定 NMIPMs 对三种物质的 动态结合曲线.

\section{3 结果与讨论}

\section{1 模板分子与功能单体之间的相互作用}

模板分子 $\mathrm{EM}$ 和功能单体 $\mathrm{MAA}$ 在聚合前是否 能形成稳定的复合物, 是获得高选择性和高亲和性 的分子印迹聚合物的关键. 因此, 本文采用紫外光 谱 ${ }^{22}$ 研究了溶液状态下不同配比 $\left(n_{\mathrm{EM}}: n_{\mathrm{MAA}}=1: 1,1: 2\right.$, $1: 4,1: 5)$ 的模板分子和功能单体之间的相互作用. 结果如图 2 所示.

由图 2 看出, $\mathrm{EM}$ 溶液的最大紫外吸收波长为 $486 \mathrm{~nm}$, 随着 MAA 加入量的增大, EM 的最大紫外 吸收向长波方向移动(红移), 且吸收峰强度增大, 表 明在氯仿中, EM与 MAA之间产生了相互作用.

为了进一步验证模板分子 $\mathrm{EM}$ 与功能单体 MAA 的相互作用情况, 将二者混合后的溶液分别 利用傅里叶变换红外光谱和核磁共振氢谱进行分 析, 其FTIR 谱图如图 3 所示.

分析可知, 在图 3(a)中, $3520 \mathrm{~cm}^{-1}$ 为 $\mathrm{OH}$ 的伸缩 


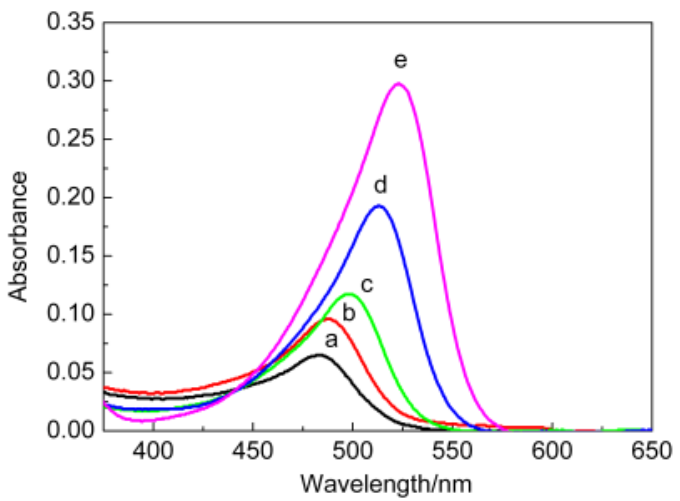

图 2 氯仿中不同摩尔比的 EM 及甲基丙烯酸(MAA) 的紫外吸收光谱

Fig.2 UV absorption spectra of EM and methacrylic acid (MAA) with different molar ratio in chloroform

(a) EM; molar ratio of EM to MAA $\left(n_{\mathrm{EM}}: n_{\mathrm{MAA}}\right)$ : (b) $1: 1$, (c) $1: 2$, (d) $1: 4$, (e) $1: 5$

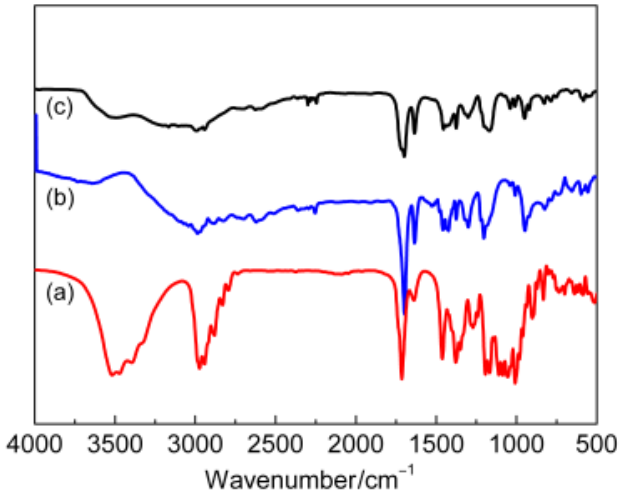

图 3 (a) EM, (b) MAA, (c) EM-MAA 的傅里叶 变换红外(FTIR)光谱图

Fig.3 Fourier transform infrared (FTIR) specta of EM (a), MAA (b), and EM-MAA (c)

振动峰, $1711 \mathrm{~cm}^{-1}$ 为 $\mathrm{C}=\mathrm{O}$ 伸缩振动峰, $1048 \mathrm{~cm}^{-1}$ 为 $\mathrm{C}-\mathrm{O}-\mathrm{C}$ 伸缩振动峰; 在图 3(b)中, $1635 \mathrm{~cm}^{-1}$ 为 $\mathrm{C}=$ $\mathrm{C}$ 双键的伸缩振动峰, $1711 \mathrm{~cm}^{-1}$ 为羧基所形成的特 征峰; 图 3(c)中, 在相同的检测浓度下, 当加入模板 分子 $\mathrm{EM}$ 后, $\mathrm{EM}$ 和 $\mathrm{MAA}$ 的 $\mathrm{C}=\mathrm{O}$ 伸缩振动峰移至 $1702 \mathrm{~cm}^{-1}$ 处, $\mathrm{EM}$ 的 $\mathrm{C}-\mathrm{O}-\mathrm{C}$ 伸缩振动峰移至 1040 $\mathrm{cm}^{-1}$ 处, 并形成了 $2310-3708 \mathrm{~cm}^{-1}$ 的宽峰, 说明 $\mathrm{EM}$ 与 MAA 确实发生了相互作用.

核磁共振氢谱如图 4 所示. 由图 4 可以看出, 在 相同的检测浓度下, 当加入模板分子 EM 后, MAA 中的羧基氢由 11.37 移至 10.47 , 再次证实模板分子 和功能单体之间产生了相互作用. 结合紫外和红外 谱图综合分析可知, EM 和 MAA之间的相互作用的 主要形式为氢键作用.

\section{2 红霉素分子印迹聚合物微球形貌和热稳定性}

采用扫描电镜对 EM-MIPMs 和 NMIPMs 进行 (a) $\mathrm{EM}$ in $\mathrm{CDCl}_{3}$
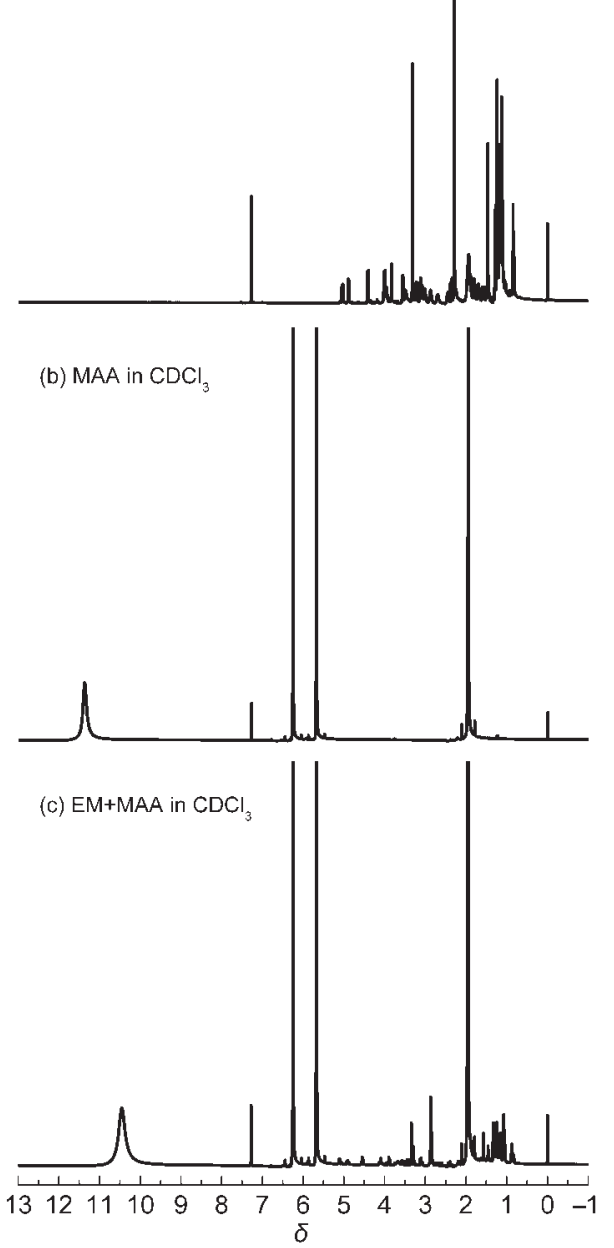

图 4 EM, MAA 和 EM-MAA 的核磁共振氢谱( ${ }^{1}$ H NMR)图

Fig.4 ${ }^{1} \mathrm{H}$ nuclear magnetic resonance (NMR) spectra of EM, MAA, and EM-MAA

微观形貌分析, 结果如图 5 所示. 由图 5 可见印迹和 非印迹微球粒径都比较均匀且颗粒规整, EMMIPMs 的粒径相对较大, 粒径分别为 4.24 和 1.16 $\mu \mathrm{m}$.

EM-MIPMs 的热重分析(TGA)曲线见图 6, 可以 看出, EM-MIPMs 在 $300{ }^{\circ} \mathrm{C}$ 以下时几乎没有热失 重, 曲线呈持平状, 表明聚合物在此范围内极其稳 定; 当温度升高到 $320-350{ }^{\circ} \mathrm{C}$ 时, 聚合物出现了失 重现象, 说明聚合物出现了部分分解的现象; 当温 度达到 $400{ }^{\circ} \mathrm{C}$ 时, 聚合物的失重率达到 $25 \%$, 说明 采用乳液聚合法制备的 EM-MIPMs 具有很好的热 稳定性.

\section{3 红霉素分子印迹聚合物微球吸附性能研究}

3.3.1 红霉素分子印迹聚合物微球的吸附等温线 目前有很多模型应用于描述吸附等温线的数 

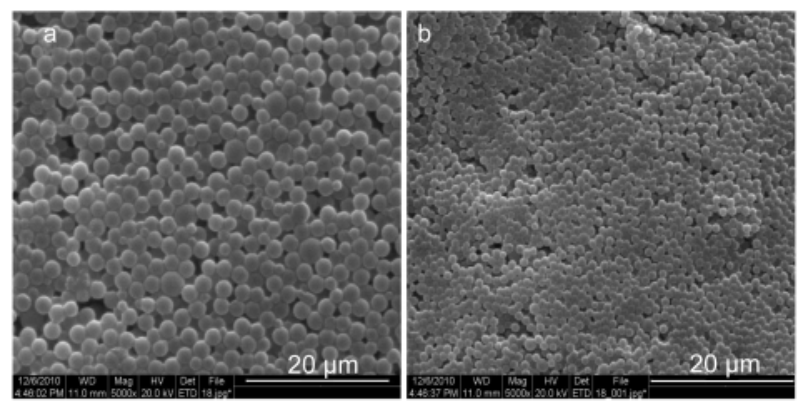

图 5 EM-MIPMs (a) 和 NMIPMs (b) 的扫描电镜(SEM) 图

Fig.5 Scanning electron microscope (SEM) images of EM-MIPMs (a) and NMIPMs (b)

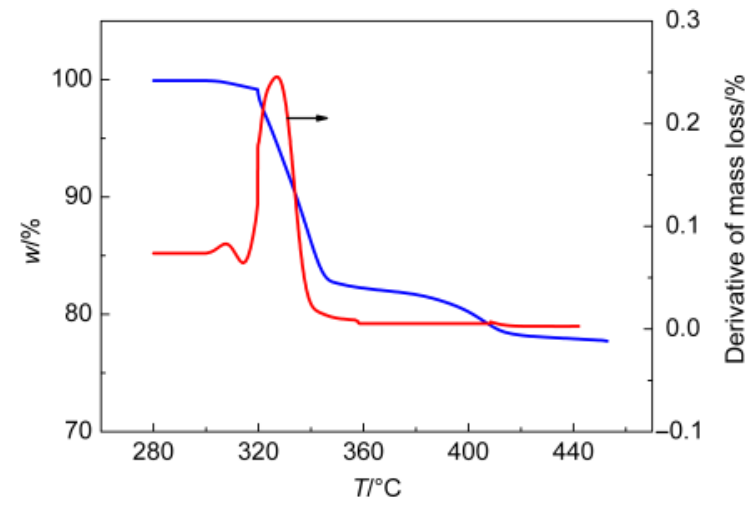

图 6 EM-MIPMs 的热重分析(TGA)曲线

Fig.6 Thermogravimetric analysis (TGA) curves of EM-MIPMs

据, 其中 Langmuir 和 Freundlich 这两组吸附等温方 程 ${ }^{23}$ 由于形式简单、参数少且容易确定而被广泛的 应用. 本文将采用这两种模型描述红霉素的吸附量 (三次平行实验的平均值)与平衡浓度之间的关系.

Freundlich吸附等温方程:

$Q_{\mathrm{e}}=K_{\mathrm{F}} C_{\mathrm{e}}{ }^{1 / n}$

式中, $K_{\mathrm{F}}$ 是最大吸附容量; $n$ 是吸附强度, 为常数.

Langmuir 吸附等温方程:

$Q_{\mathrm{e}}=K_{\mathrm{L}} Q_{\mathrm{m}} C_{\mathrm{e}} /\left(1+K_{\mathrm{L}} C_{\mathrm{e}}\right)$

式中, $C_{\mathrm{e}}$ 是红霉素的平衡浓度 $\left(\mathrm{mmol} \cdot \mathrm{L}^{-1}\right) ; Q_{\mathrm{e}}$ 是吸附 容量 $\left(\mathrm{mmol} \cdot \mathrm{g}^{-1}\right) ; K_{\mathrm{L}}$ 是吸附分配系数 $\left(\mathrm{L} \cdot \mathrm{mmol}^{-1}\right) ; Q_{\mathrm{m}}$ 是最大吸附容量 $\left(\mathrm{mmol} \cdot \mathrm{g}^{-1}\right)$.

Langmuir 吸附等温线的本质特征可以由一个

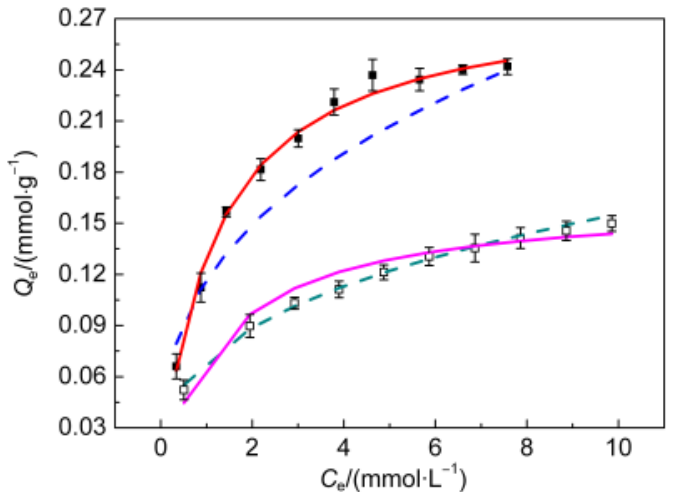

图 $725^{\circ} \mathrm{C}$ 时 EM-MIPMs 和 NMIPMs 吸附 EM 的 非线性 Langumir 和 Freundlich 等温吸附线

Fig.7 Nonlinear isotherms of Langumir and Freundlich for the adsoption of EM onto EM-MIPMs and NMIPMs at $25^{\circ} \mathrm{C}$

) static adsorption experimental value of EM-MIPMs; ( $\square$ ) static adsorption experimental value of NMIPMs; (-) Langumir isotherms of EM-MIPMs; (---) Freundlich isotherms of EM-MIPMs; (一) Langumir isotherms of NMIPMs; (---) Freundlich isotherms of NMIPMs

平衡参数 $R_{\mathrm{L}}$ 表示, 其计算公式为 $:^{24}$

$$
R_{\mathrm{L}}=1 /\left(1+C_{\mathrm{m}} K_{\mathrm{L}}\right)
$$

式中, $C_{\mathrm{m}}$ 是 $\mathrm{EM}$ 的最大初始浓度 $\left(\mathrm{mmol} \cdot \mathrm{L}^{-1}\right)$. 平衡参 数 $R_{\mathrm{L}}$ 有四种可能值: (1) $0<R_{\mathrm{L}}<1.0$, 表示吸附条件适 宜; (2) $R_{\mathrm{L}}>1.0$, 表示吸附条件不适宜; (3) $R_{\mathrm{L}}=1.0$, 表 示为线性吸附; (4) $R_{\mathrm{L}}=0$, 表示为不可逆吸附.

EM-MIPMs 和 NMIPMs 的吸附等温线及相关 参数如图 7 和表 1 所示。

由图 7 可看出随着 EM 浓度的增大 EM-MIPMs 和 NMIPMs 的吸附量也在增加, 饱和吸附量分别为 0.242 和 $0.149 \mathrm{mmol} \cdot \mathrm{g}^{-1}$. 在相同浓度下单位质量的 EM-MIPMs 对 EM 的吸附量明显高于 NMIPMs 的吸 附量, 印迹因子为 1.62, 表明 EM-MIPMs 对 EM 有着 显著的吸附效果. ${ }^{25}$ 这种现象说明在印迹过程中, 模 板分子 EM 与功能单体 MAA之间通过相互作用在 EM-MIPMs 上产生了印迹孔穴及印迹位点, 决定了 EM-MIPMs 对 EM 的高度亲合力和特异识别性. 依

表 $125^{\circ} \mathrm{C}$ 时 EM-MIPMs 和 NMIPMs 吸附 EM 的 Langmuir 与 Freundlich 模型参数

Table 1 Langmuir and Freundlich model parameters for adsorption of EM onto EM-MIPMs and NMIPMs at $25^{\circ} \mathrm{C}$

\begin{tabular}{|c|c|c|c|c|c|c|c|}
\hline \multirow{2}{*}{ Adsorbent } & \multicolumn{4}{|c|}{ Langmuir } & \multicolumn{3}{|c|}{ Freundlich } \\
\hline & $K_{\mathrm{L}} /\left(\mathrm{L} \cdot \mathrm{mmol}^{-1}\right)$ & $Q_{\mathrm{m}} /\left(\mathrm{mmol} \cdot \mathrm{g}^{-1}\right)$ & $R^{2}$ & $R_{\mathrm{L}}$ & $K_{\mathrm{F}} /\left(\mu \mathrm{mol} \cdot \mathrm{g}^{-1}\right)$ & $n /\left(\mathrm{L} \cdot \mu \mathrm{mol}^{-1}\right)$ & $R^{2}$ \\
\hline EM-MIPMs & 0.846 & 0.284 & 0.997 & 0.106 & 9.92 & 2.87 & 0.934 \\
\hline NMIPMs & 0.794 & 0.160 & 0.983 & 0.112 & 10.4 & 3.35 & 0.996 \\
\hline
\end{tabular}

$K_{\mathrm{L}}$ : Langmuir equilibrium constant; $Q_{\mathrm{m}}$ : maximum adsorption capacity according to Langmuir monolayer adsorption; $R_{\mathrm{L}}$ : Langmuir equilibrium parameter; $K_{\mathrm{F}}$ : Freundlich constant related to adsorption capacity of the adsorbent; $n$ : Freundlich constant related to adsorption intensity of the adsorbent; $R^{2}$ : correlation coefficient 
靠 EM-MIPMs 与 EM 分子之间非共价作用, 这种印 迹孔穴和印迹位点就会对 EM产生较强的吸附作 用, 而 NMIPMs 表面没有印迹孔穴和印迹位点, 只 限于微球表面产生的非特异性吸附, 因此吸附量较 低.

经计算静态平衡吸附数据, 使用 Langumir 与 Freundlich 两种等温吸附方程进行分析, 分析结果如 表1所示.

由表 1 以及图 7 可以明显看到, 在 $25^{\circ} \mathrm{C}$ 时, $\mathrm{EM}$ 在 EM-MIPMs 上的吸附行为更好地符合 Langmuir 等温吸附方程, 其方程为 $C_{\mathrm{e}} / Q_{\mathrm{e}}=0.58+6.26 C_{\mathrm{e}}$. 由 Langmuir 等温吸附方程可知, EM 在 EM-MIPMs 上 是以单分子层的形式吸附于微球表面的, 理论最大 吸附容量为 $0.284 \mathrm{mmol} \cdot \mathrm{L}^{-1}$, 远高于 NMIPMs $\left(0.160 \mathrm{mmol} \cdot \mathrm{L}^{-1}\right)$. 吸附容量的较大差异是因为 $\mathrm{EM}$ MIPMs 上分布有很多与模板分子结构相匹配的孔 穴和印迹位点, 这使得模板分子能够很好地结合到 EM-MIPMs 上. Ferundlich 等温吸附方程是一个经验 公式, 它主要基于多层吸附而产生的指数增长模 型, 由表 1 和图 6 可以看出, EM 在 NMIPMs 上的吸 附行为更好地符合 Ferundlich 等温吸附方程, 这表 明 NMIPMs 对 EM 的吸附主要是多层吸附. 此外表 1 中 $R_{\mathrm{L}}$ 值(介于 0 到 1.0 之间)和 $n$ (大于 1.0 )表明吸附实 验条件很适合 $\mathrm{EM}$ 的吸附. ${ }^{26}$

\subsection{2 红霉素分子印迹聚合物微球吸附动力学 研究}

吸附动力学是研究分子印迹聚合物达到平衡 所需的时间和吸附速率的有效方法, 本文通过准一 级动力学方程和准二级动力学方程来研究 EMMIPMs 对 EM 的吸附动力学. 吸附动力学曲线如图 8 所示.

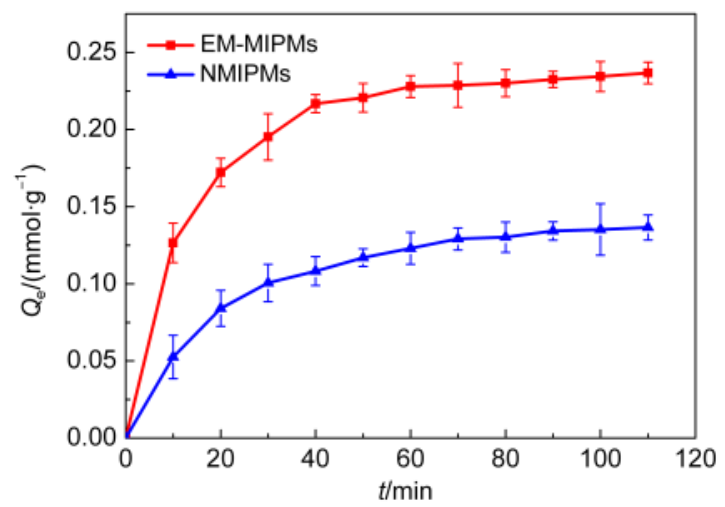

图 8 EM-MIPMs 和 NMIPs 的吸附动力学曲线

Fig.8 Curves of adsorption dynamics of EM-MIPMs and NMIPMs
由图 8 可以看出, EM-MIPMs 在前 $40 \mathrm{~min}$ 吸附 量迅速增加, 这段时间主要发生的是 EM-MIPMs 外 表面对 EM 的吸附, 吸附量占总量的 $84 \% ; 40-70$ $\min$ 吸附量增加变缓, 此时主要发生 EM 通过孔道向 内表面扩散后的吸附, 吸附量占总量的 $97 \%$; 至 80 $\min$ 时吸附已基本达到饱和. 整个吸附过程出现的 现象表明 EM-MIPMs 中由交联剂和功能单体构成 的立体孔穴分布深浅不一, 在开始阶段由于聚合物 微球外表面分布着大量印迹位点, 因而对模板分子 的表现快速结合; 随着外表面结合逐渐达到饱和, EM 开始向深孔传质, 此时会有一定的空间位阻效 应, 导致吸附速率显著下降, 最终直至饱和. EMMIPMs 对 EM 的吸附在 80 min 已基本达到饱和, 体 现了对 EM 的快速响应能力.

准一级动力学方程和准二级动力学方程如(5)、 (6)式所示: ${ }^{27,28}$

$$
\begin{aligned}
& \ln \left(Q_{\mathrm{e}}-Q_{t}\right)=\ln Q_{\mathrm{e}}-k_{1} t \\
& t / Q_{t}=1 /\left(k_{2} Q_{\mathrm{e}}^{2}\right)+t / Q_{\mathrm{e}}
\end{aligned}
$$

其中 $Q_{\mathrm{e}}$ 为平衡吸附量 $\left(\mathrm{mmol} \cdot \mathrm{g}^{-1}\right), Q_{t}$ 为 $t$ 时刻的吸附 量 $\left(\mathrm{mmol} \cdot \mathrm{g}^{-1}\right), k_{1}$ 是准一级动力学方程吸附速率常数 $\left(\mathrm{min}^{-1}\right), k_{2}$ 是准二级动力学方程吸附速率常数 $(\mathrm{g}$. $\mathrm{mmol}^{-1} \cdot \mathrm{min}^{-1}$ ). 使用的数据为三次平行实验的平均 值.

准一级、准二级动力学方程的拟合曲线如图 9 所示, 动力学拟合曲线参数见表 2 .

由图 9 和表 2 可知, EM-MIPMs 和 NMIPMs 与 准二级动力学方程相关性优于准一级动力学方程. EM-MIPMs 的准二级动力学方程为 $t / Q_{t}=0.0349+$ $0.004 t$, 与准二级动力学方程线性相关性均为 0.999 , 且计算平衡吸附量与实验平衡吸附量相差较小, 说 明准二级动力学方程可以更好的描述吸附过程, 化 学吸附过程是控制 EM吸附识别的主要过程, 由表 2 还可以看出, EM-MIPMs 的 $k_{2}$ 值远大于 NMIPMs 的 $k_{2}$ 值, 这表明 EM-MIPMs 不仅在吸附容量和强度上 优于 NMIPMs, 而且在吸附速率上远大于 NMIPMs. ${ }^{29}$

\section{4 红霉素分子印迹聚合物微球的识别选择性}

图 10 为 EM-MIPMs 和 NMIPMs 对 EM, RM 和 EEs 的动态结合曲线.

从图 10(a)可以看到 RM 和 EEs 溶液的泄漏体积 分别是 16 和 $8 \mathrm{BV}$, 而 $\mathrm{EM}$ 溶液的泄露体积表现出较 高数值 (62 BV), 经计算发现, EM-MIPMs 对 RM 的 泄漏吸附量与饱和吸附量仅为 0.0296 和 0.0593 $\mathrm{mmol} \cdot \mathrm{g}^{-1}$, 对 $\mathrm{EEs}$ 的泄漏吸附量与饱和吸附量为 

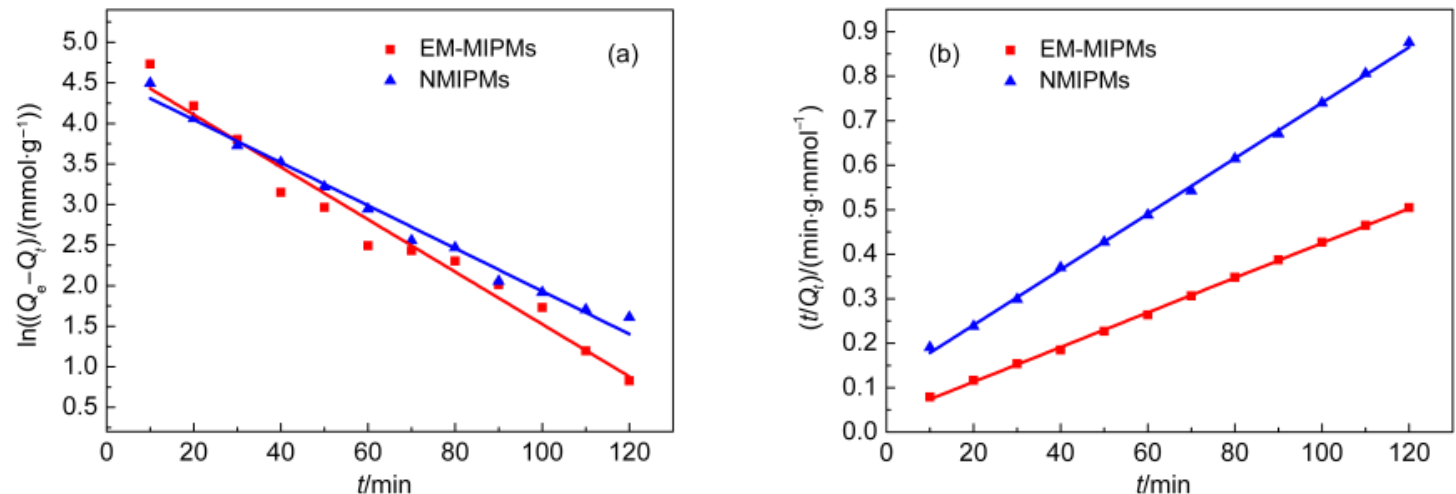

图 $925^{\circ} \mathrm{C}$ 时 EM-MIPMs 和 NMIPMs 的准一级(a)和准二级(b)动力学模型

Fig.9 Pseudo-first-order kinetics (a) and pseudo-second-order kinetics (b) models of EM-MIPMs and NMIPMs at $25^{\circ} \mathrm{C}$

表 $225^{\circ} \mathrm{C}$ 时准一级和准二级模型的动力学参数

Table 2 Kinetic constants for the pseudo-first-order and pseudo-second-order models at $25^{\circ} \mathrm{C}$

\begin{tabular}{|c|c|c|c|c|c|c|c|}
\hline \multirow{2}{*}{ Adsorbent } & \multirow{2}{*}{$Q_{\mathrm{c}, \mathrm{exp}} /\left(\mathrm{mmol} \cdot \mathrm{g}^{-1}\right)$} & \multicolumn{3}{|c|}{ Pseudo-first-order } & \multicolumn{3}{|c|}{ Pseudo-second-order } \\
\hline & & $k_{1} / \mathrm{min}^{-1}$ & $Q_{\mathrm{e}, \mathrm{ca}} /\left(\mathrm{mmol} \cdot \mathrm{g}^{-1}\right)$ & $R^{2}$ & $k_{2} /\left(\mathrm{g} \cdot \mathrm{mmol}^{-1} \cdot \mathrm{min}^{-1}\right)$ & $Q_{\mathrm{e}, \mathrm{ca}} /\left(\mathrm{mmol} \cdot \mathrm{g}^{-1}\right)$ & $R^{2}$ \\
\hline EM-MIPMs & 0.240 & 0.0322 & 0.116 & 0.969 & 43.6 & 0.256 & 0.999 \\
\hline NMIPMs & 0.142 & 0.0258 & 0.0974 & 0.986 & 0.337 & 0.160 & 0.999 \\
\hline
\end{tabular}

$Q_{c, \text { cxp }}:$ equilibrium adsorption experimental value; $Q_{\text {c,cal }}$ : equilibrium adsorption calculated value; $k_{1}$ : adsorption rate constant of Pseudo-first-order kinetics model; $k_{2}$ : adsorption rate constant of Pseudo-second-order kinetics model
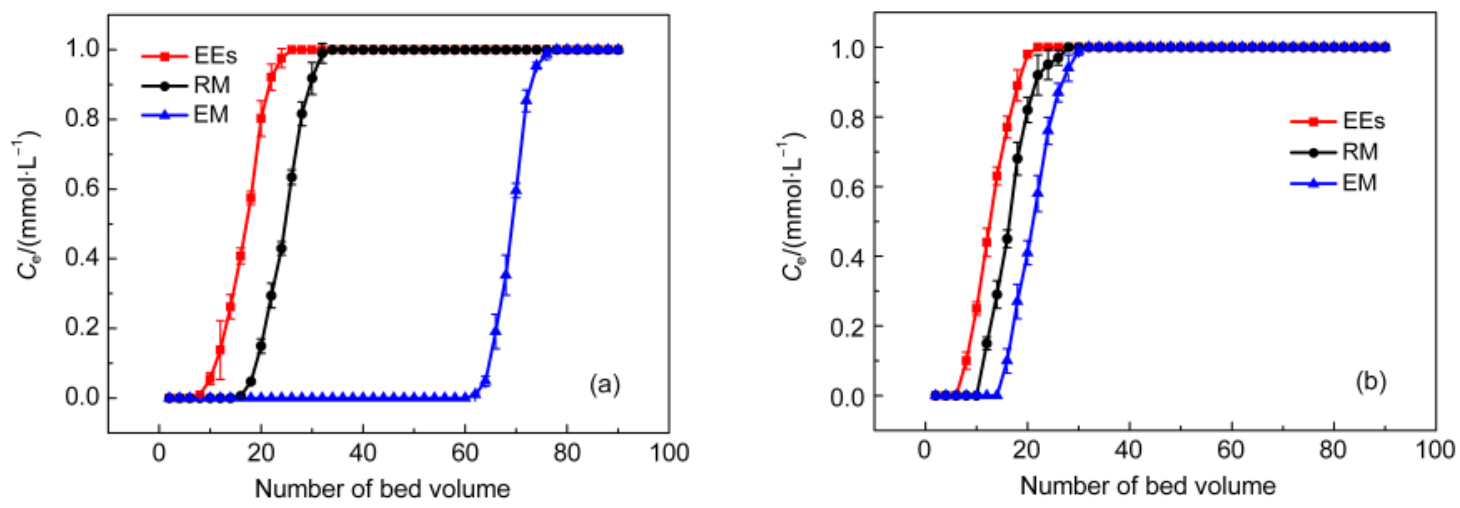

图 10 EM-MIPMs (a)和 NMIPMs (b) 对 EM, RM 和 EEs 的动态结合曲线

Fig.10 Dynamic adsorption curves of EM-MIPMs (a) and NMIPMs (b) for EM, RM, and EEs bed volume (BV): $2 \mathrm{~mL}$; temperature: $25^{\circ} \mathrm{C}$; initial concentration: $1 \mathrm{mmol} \cdot \mathrm{L}^{-1}$; flow rate: $2 \mathrm{BV} \cdot \mathrm{h}^{-1}$

0.0148 和 $0.0444 \mathrm{mmol} \cdot \mathrm{g}^{-1}$, 而对 $\mathrm{EM}$ 的泄漏吸附量 与饱和吸附量则高达 0.115 和 $0.141 \mathrm{mmol} \cdot \mathrm{g}^{-1}$. 由这 些数据可以看出 EM-MIPMs 对 EEs 和 RM 的吸附量 明显小于对 EM 的吸附量. 这是由于 EM-MIPMs 上 具有和 EM 尺寸及空间构型一致的孔穴, 因而模板 分子 EM 能够进去孔穴之中而表现出较强的保留, 但对其它两种竞争物质基本不选择、不识别、不结 合, 该结果表明 EM-MIPMs 的特异性识别能力较 好.

从图 10(b) 中可以看出, NMIPMs 作为填充柱 时, 对 EM、RM 和 EEs 三种大环内酯类抗生素溶液 的泄漏体积相差较小, 分别为 $18 、 12$ 和 $8 \mathrm{BV}$. 这是由
于这三种大环内酯类物质的结构十分的相似, NMIPMs 对三者的吸附都属于物理吸附, 因而没有 什么明显差别.

\section{4 结 论}

以 $\mathrm{EM}$ 为模板分子，采用乳液聚合法制备了 EM-MIPMs, 研究发现, 由于 EM-MIPMs 表面上分 布有大量 EM 印迹位点, 使得 EM-MIPMs 对 EM 具 有优良的结合性能, 且主要为单分子层吸附, 饱和 吸附量为 $0.242 \mathrm{mmol} \cdot \mathrm{g}^{-1}$. EM-MIPMs 对 EM 的吸 附速率符合准二级动力学方程, 表明主要控速步骤 为化学吸附. 在 EM 以及与其结构相似的抗生素 
EEs 及 $R M$ 作为竞争底物的体系中, EM-MIPMs 对 EM 具有高度的选择识别性, 且 EM-MIPMs 具有结 构规整、粒径均匀, 热稳定性良好的性质.

\section{References}

(1) Scorrano, S.; Longo, L.; Vasapollo, G. Anal. Chim. Acta 2010, 659, 167. doi: 10.1016/j.aca.2009.11.046

(2) Zhang, Z. D.; Zheng, S. J.; Yang, J.; Wang, W. M.; Liu, B. Z.; Zhu, X. L. Chinese Journal of Chemical Physics 2013, 26 (3), 361. [张志丹, 郑赛晶, 杨 俊, 王维妙, 刘百战, 朱晓兰. 化学 物理学报, 2013, 26 (3), 361.] doi: 10.1063/1674-0068/26/03/ 361-368

(3) Geng, L. N.; Kang, N.; Ning, Z. Y.; Wu, L. Q.; Li, Y.; Sun, L. Q.; Luo, A. Q. Acta Phys. -Chim. Sin. 2008, 24 (1), 25. [耿利 娜, 康 宁, 宁周云, 武利庆, 李 琰, 孙立权, 罗爱芹. 物理化 学学报, 2008, 24 (1), 25.] doi: 10.3866/PKU.WHXB20080105

(4) Qiu, Z. Y.; Zhong, S. A. Acta Chim. Sin. 2010, 68 (3), 246. [邱 增英, 钟世安. 化学学报, 2010, 68 (3), 246.]

(5) Huang, Y. H.; Xu, Y.; Du, B. B.; He, Q. H.; Cao, Y. S. Chin. J. Anal. Chem. 2012, 40 (7), 1011. [黄运红, 许 扬, 杜碧柏, 何 庆华, 曹郁生. 分析化学, 2012, 40 (7), 1011.] doi: 10.1016/ S1872-2040(11)60558-6

(6) Chen, Z. D.; Wei, J. X.; Wang, W. C.; Kong, Y. Chin. Chem. Lett. 2010, 21 (3), 353.

(7) Curcio, M.; Puoci, F.; Cirillo, G.; Iemma, F.; Spizzirri, U. G.; Picci, N. J. Agric. Food Chem. 2010, 58 (22), 11883. doi: $10.1021 / \mathrm{jf102936p}$

(8) Sellergren, B.; Karmalkar, R. N.; Shea, K. J. J. Org. Chem. 2000, 65 (13), 4009. doi: 10.1021/jo000014n

(9) Men, J. Y.; Gao, B. J.; Chen, Z. P.; Yao, L. Acta Chim. Sin. 2012, 70 (21), 2273. [门吉英, 高保娇, 陈志萍, 么 兰. 化学学报, 2012, 70 (21), 2273.] doi: 10.6023/A12070402

(10) Ornelasa, M.; Loureiroa, D.; Araújoa, M. J.; Marquesa, E.; DiasCabralb, C.; Azenhaa, M.; Silva, F. J. Chromatogr. A 2013, 1297 (5), 138.

(11) Dvorakova, G.; Haschick, R.; Chiad, K.; Klapper, M.; Müllen, K.; Biffis, A. Macromol. Rapid Commun. 2010, 31 (23), 2035. doi: 10.1002/marc.201000406

(12) Xu, Z. F.; Wen, G.; Kuang, D. Z.; Zhang, F. X.; Tang, S. P. J. Environ. Sci. Heal. B 2013, 48 (5), 336. doi: 10.1080/ 03601234.2013.742372

(13) Li, J. J.; Yang, M.; Huo, D. Q.; Hou, C. J.; Li, X. L.; Wang, G. M.; Feng, D. J. Sep. Sci. 2013, 36 (6), 1142. doi: 10.1002/ jssc. 201200869
(14) Dai, J. D.; Pan, J. M.; Xu, L. C.; Li, X. X.; Zhou, Z. P.; Zhang, R. X.; Yan, Y. S. J. Hazard. Mater. 2012, 205-206, 179.

(15) Rodríguez, E.; Navarro-Villoslada, F.; Benito-Peñña, E.; Marazuela, M. D.; Moreno-Bondi, M. C. Anal. Chem. 2011, 83 (6), 2046. doi: 10.1021/ac102839n

(16) He, J.; Tang, H. Z.; You, L. Q.; Yuan, L. L.; Liu, Z. M.; Zhu, J.; Lu, K.; Chen, X. Z. Chromatographia 2013, 76 (15-16), 959.

(17) Geng, L. Y.; Kou, X.; Lei, J. D.; Su, H. J.; Ma, G. H.; Su, Z. G. J. Chem. Technol. Biot. 2012, 87 (5), 635. doi: 10.1002/jctb. $\mathrm{v} 87.5$

(18) Kou, X.; Geng, L. Y.; Lei, J. D.; Ma, G. H.; Su, Z. G. The Chinese Journal of Process Engineering 2011, 11 (3), 481. [寇 星, 耿立媛, 雷建都, 马光辉, 苏志国. 过程工程学报, 2011, 11 (3), 481.]

(19) Guan, P.; Hu, X. L.; Hao, M. Y. J. Funct. Mater. 2010, 41 (z2), 379. [管 萍, 胡小玲, 郝明燕. 功能材料, 2010, 41 (z2), 379.]

(20) Hu, X. L.; Guan, P.; Zhu, L.; Zhang, X. L. Preparation Method of Macrolide Antibiotics Molecularly Imprinted Polymer Microspheres. CN Patent 101507916A, 2009-08-19. [胡小玲, 管 萍, 朱 丽, 张新丽. 大环内酯类抗生素分子印迹聚合物 微球的制备方法. 中国: CN101507916A[P], 2009-08-19.]

(21) Song, R. Y.; Hu, X. L.; Guan, P.; Yin, Y. N.; Zhu, L. Ion Exchange and Adsorption 2013, 29 (2), 97 . [宋任远, 胡小玲, 管 萍, 尹亚楠, 朱 丽. 离子交换与吸附, 2013, 29 (2), 97. ]

(22) Andersson, H. S.; Nicholls, I. A. Bioorg. Chem. 1997, 25 (3), 203. doi: 10.1006/bioo.1997.1067

(23) Li, Q.; Yue, Q. Y.; Su, Y.; Gao, B. Y.; Sun, H. J. Chem. Eng. J. 2010, 158 (3), 489. doi: 10.1016/j.cej.2010.01.033

(24) Greluk, M.; Hubicki, Z. Chem. Eng. J. 2010, 162 (3), 919. doi: 10.1016/j.cej.2010.06.043

(25) Pan, J. M.; Yao, H.; Xu, L. C.; Ou, H. X.; Huo, P. W.; Li, X. X.; Yan, Y. S. J. Phys. Chem. C 2011, 115 (13), 5440. doi: 10.1021/ jp111120x

(26) Li, X. X.; Pan, J. M.; Dai, J. D.; Dai, X. H.; Xu, L. C.; Wei, X.; Hang, H.; Li, C. X.; Liu, Y. Chem. Eng. J. 2012, 198-199, 503.

(27) Abramian, L.; El-Rassy, H. Chem. Eng. J. 2009, 150 (2-3), 403. doi: 10.1016/j.cej.2009.01.019

(28) Zhang, Z. Z.; Zhang, Z. B.; Fernández, Y.; Menénde, J. A.; Niu, H.; Peng, J. H.; Zhang, L. B.; Guo, S. H. Appl. Surf. Sci. 2010, 256 (8), 2569. doi: 10.1016/j.apsusc.2009.10.106

(29) Zhou, L. C.; Meng, X. G.; Li, J. M.; Hu, W.; Liu, B.; Du, J. Acta Phys. -Chim. Sin. 2012, 28 (7), 1615. [周良春, 孟祥光, 李建 梅, 胡 伟, 刘 波, 杜 娟. 物理化学学报, 2012, 28 (7), 1615.] doi: 10.3866/PKU.WHXB201204282 